\title{
A Role for SEM in Autopsy Cases of TRALI
}

\author{
Patricia E. Kysar, Grete N. Adamson and Hanne M. Jensen
}

Department of Pathology and Laboratory Medicine, U.C. Davis, Davis, California

H. M. Jensen et al. (2010) [1] investigated three autopsy cases from our hospital with deaths due to transfusion-related acute lung injury (TRALI) [2]. Common features of the lungs by light microscopy (LM) stained with hematoxylin and eosin (H\&E) showed congestion of capillaries and venules, focal aggregates of macrophages, fluid in alveoli, and, in one case intravascular dark material. Under polarized light, the dark material was birefringent. To elucidate the nature of the material, scanning electron microscopy (SEM) was performed. Pulmonary tissues stored in fixative were standardly prepared [3] and viewed by SEM. Correlation between the LM and SEM would be important but a standard tissue block did not allow correlation. The standard protocol with critical point drying did not maintain the dark/birefringent material. Infiltration with hexamethyldisilazane (HMDS) [4] and evaporation was done by Abela and Aziz [5] to maintain cholesterol in their samples.

M.A. Hayat [3] discussed the use of paraffin extraction from thick sections in 1978 for SEM. By using adjoining sections, one for LM and a thicker one for SEM using HMDS we could look at the same venule and discover the nature of the dark/birefringent material. Coordinates utilized by Geissinger et al.[6] would not be required. The success of finding the same venule in both modalities relied heavily on the unique structure of the pulmonary tissue.

Autopsied lung tissues fixed in 10\% formalin were prepared utilizing a standard protocol including critical point drying; Samdri pvt-3, Tousimis, Rockville, Md. and viewed in the SEM. Tissue was also processed into paraffin. Sections were cut from the blocks at two different thicknesses: $4 \mu \mathrm{m}$ for LM and $10 \mu \mathrm{m}$ for SEM. Our service lab placed individual sections on glass slides. SEM sections were de-paraffinized in $3 \times 10 \mathrm{~min}$ changes of $100 \%$ xylene, dehydrated with 2 X10min changes in $100 \%$ ethyl alcohol (ETOH) and infiltrated with hexamethyl-disilazane (HMDS) beginning with a 10min 1:1 followed by 3 X15min changes of 100\% HMDS and overnight evaporation in a fumehood. Each slide was scored and broken to fit a $32 \mathrm{~mm}$ aluminum stub and mounted with double-stick carbon tape. Samples were gold-coated and viewed on a Philips XL30 scanning electron microscope.

The H\&E sections were viewed and photographed for orientation using an Olympus BH-2 light microscope and Spot Insight2 Color Camera. A specific area of interest on the H\&E section was selected for structure and birefringence and micrographed as seen in Fig. A. The arrows indicate a venule with dark/birefrigent material. The initial preparation using the critical point dryer demonstrated good preservation but there was no indication of any material lining the venules [fig.B]. Abbreviated dehydration in ETOH and HMDS infiltration with subsequent evaporation maintained the material indicated by the arrows in Fig. C. Fig. C shows the same venule as seen in Fig. A. Fig.D, at higher magnification, shows the crystalline nature of the material confirmed as cholesterol [1] with its pronounced angularity. One sharp point is indicated by an arrow.

This unconventional approach to study an autopsy case was productive. It allowed viewing in both the LM and SEM to compare the condition of pulmonary venules and a substance lining them. SEM images of the sharp crystalline nature of the material contributed to an explanation of the cause of death in cases of TRALI [1]. The standard SEM preparation protocol for a tissue block processed according to Hayat [3] yielded excellent ultrastructure with no sign of the dark/birefringent material was seen in LM. Changing the protocol to a short ascending concentration of ETOH, using HMDS [4] as was done by Abala and Aziz [5] in their investigation of cholesterol was essential. Viewing consecutive sections for LM and in SEM allowed us to investigate the same venule with the dark/birefringent material. Finding the same pulmonary venule in the SEM was simple with the aid of a digital image of the H\&E section. A painstaking comparison of the coordinates in the LM and SEM as had been done by Geissinger et al. [6] was not necessary. The analysis in Jensen H.M. et al [1] by gas chromatography-mass spectroscopy (GC-MS) confirmed the presence of cholesterol in the autopsy tissue. We assume the dark/birefrigent material seen in the LM and the corresponding aggregations along venules in the SEM is cholesterol. 


\section{References:}

[1] Jensen et al. (2010) TRALI is due to pulmonary venule damage from leucocytes with cholesterol crystal formation. Vox Sanuinis 98, 130-137.

[2] Popovshy M.A. et al. (1983) Transfusion-related acute lung injury associated with passive transfer of anti-leukocyte antibodies. Am. Rev. Respir. Dis. 128:185-189.

[3] Hayat, M.A. (1978) Introduction to Biological Scanning Electron Microscopy. University Park Press, Baltimore, Md.

[4] Nation, J.L. (1983) A new method using hexamethyldisilazane for preparation of soft insect tissues for scanning electron microscopy. Biotech. Histochem. 58:347-351.

[5] Abela, G.S. and Aziz, K. (2006) Cholesterol crystals rupture biological membranes and human plaques during acute cardiovascular events- A novel insight into plaque rupture by scanning electron microscopy. Scanning 28, 1-10.

[6] Geissinger et al. (1973) Fast scanning electron microscopic and light microscopic correlation of paraffin sections and This work was supported by a grant awarded to H. M. Jensen from the Department of Pathology and Laboratory Medicine, University of California, Davis Medical Center, Sacramento, CA, 95816

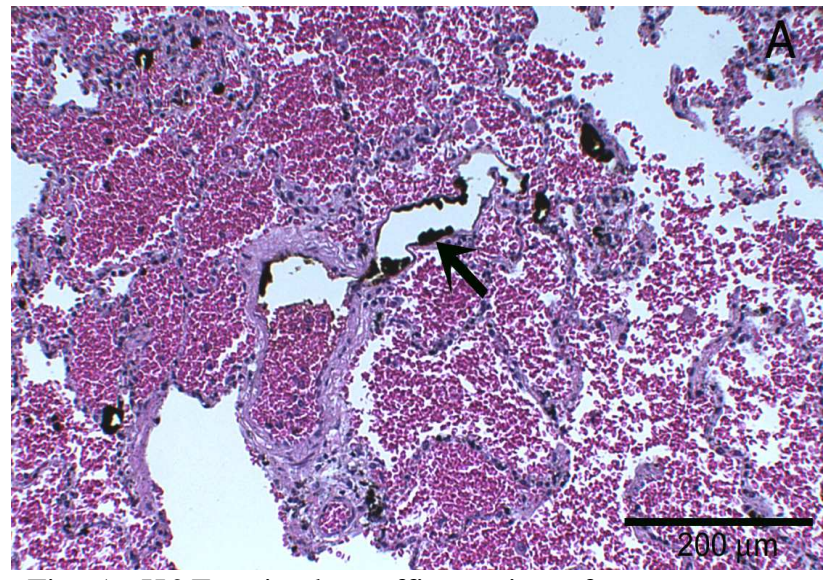

Fig. A: H\&E stained paraffin section of lung showing dark material in a vessel (arrow).

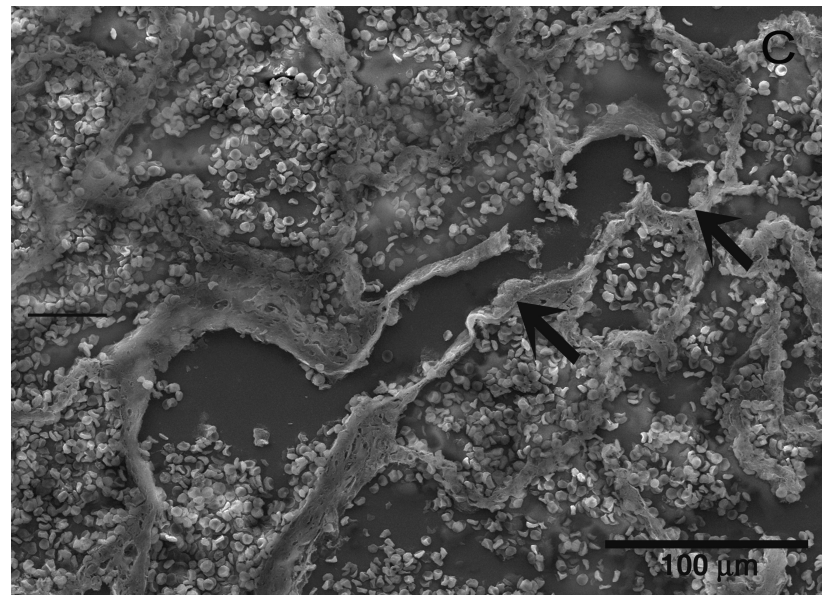

Fig. C: SEM image of the vessel containing dark material (arrows).

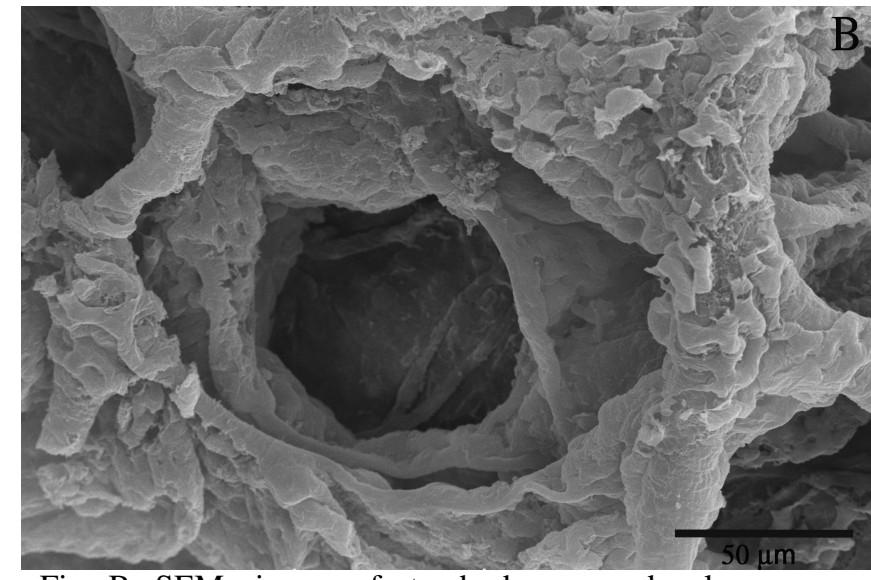

Fig. B: SEM image of standard-processed pulmonary tissue. No orientation was possible. No material was seen in venules.

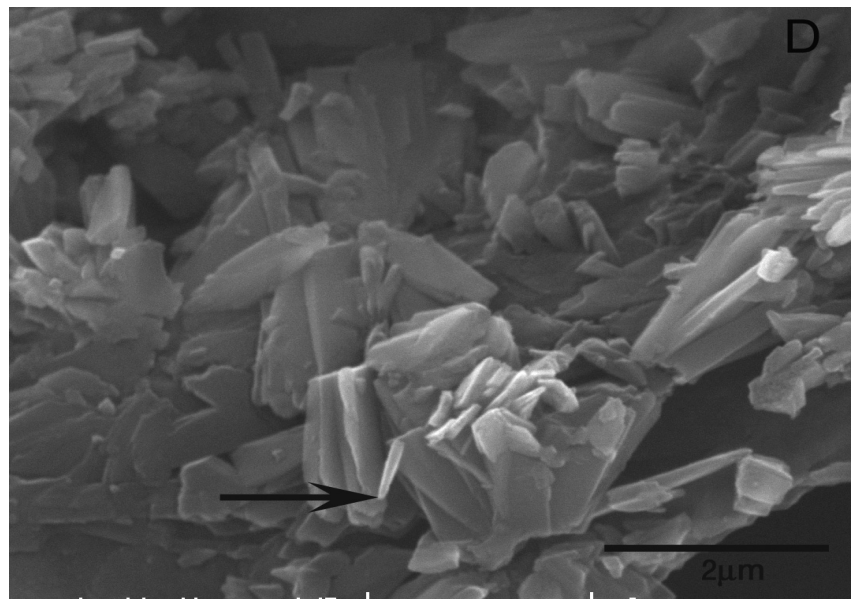

Fig D: At higher magnification of the dark material, the crystalline structure can be appreciated.. The arrow shows a sharp point. 